\title{
Maximal kidney length predicts need for native nephrectomy in ADPKD patients undergoing renal transplantation
}

\author{
Octav Cristea, MD; ${ }^{*}$ Daniel Yanko, MD; ${ }_{i}^{+}$Sarah Felbel, MD; ${ }^{\dagger}$ Andrew House, MD; ${ }^{\ddagger}$ Alp Sener, MD, FRCSC,; ${ }^{* \neq}{ }^{*}$ \\ Patrick P.W. Luke, MD, FRCSC ${ }^{* \neq \xi}$
}

*Schulich School of Medicine \& Dentistry, Western University, London, ON; 'Department of Surgery, Division of Urology, Western University, London, ON; ${ }^{*}$ Multi-Organ Transplant Program, London Health Sciences Centre, Western University, London, ON; §Matthew Mailing Centre for Translational Transplant Studies, London Health Sciences Centre, Western University, London, ON; \pm Department of Medicine, Division of Nephrology, Western University, London, ON

Cite as: Can Urol Assoc J 2014;8(7-8):278-82. http://dx.doi.org/10.5489/cuaj.2128 Published online August 11, 2014.

\section{Abstract}

Introduction: Native nephrectomy in patients with autosomal dominant polycystic kidney disease (ADPKD) is performed on a case-by-case basis. We determine if pre-transplant maximal kidney length (MKL) can be used to predict ultimate nephrectomy status. Methods: We performed a retrospective review of ADPKD patients who underwent renal transplantation at our centre between January 2000 and December 2012. Pre-transplant measurements of MKL alone, MKL adjusted for height (HtMKL), weight (WtMKL) and body mass index (BMI-MKL) were each assessed for their predictive ability via a receiver operating characteristic (ROC) curve analysis.

Results: In total, 84 patients met our inclusion criteria, of which 17 (20.2\%) underwent native nephrectomy. An MKL ROC curve analysis revealed an area under the curve (AUC) of 0.867 (95\% confidence interval [CI] $0.775-0.931 ; p<0.001)$. An optimal cutoff criterion of $>21.5 \mathrm{~cm}$ revealed a sensitivity of $94.1 \%(95 \%$ $\mathrm{Cl} 71.3-99.9)$ and specificity of $70.1 \%(95 \% \mathrm{Cl} 57.7-80.7)$ for eventual nephrectomy. The AUC of HtMKL, WtMKL and BMI-MKL ROC curves did not differ significantly from $M K L$ alone. HtMKL improved specificity, but not overall test performance. The determination of the cut-off MKL may be influenced by the single-centre retrospective nature of this analysis, as well as the fact that renal size was determined by ultrasound and not computerized tomography or magnetic resonance imaging.

Conclusion: MKL in patients with ADPKD is associated with the eventual need for nephrectomy and may be a useful clinical tool to risk stratify these patients and therefore guide patient conversations to a decision to leave the native kidneys in situ.

\section{Introduction}

Autosomal dominant polycystic kidney disease (ADPKD) is the most common hereditary disorder of the kidneys, with a worldwide prevalence ranging from $1: 400$ to $1: 1000 .^{1}$ It is primarily characterized by the gradual development of cysts within the kidney, which over time result in renal parenchymal loss and the development of end-stage renal disease (ESRD) in a substantial proportion of sufferers. ${ }^{2,3}$ Although the rate of cystic growth and accompanying kidney enlargement varies between individuals, there is a positive trend over time. ${ }^{4,5}$ Increases in total kidney volume (TKV) precede and reliably predict declining glomerular function, ${ }^{6,7}$ such that by the time that they develop ESRD most patients likely have markedly enlarged kidneys.?

Many patients suffer symptomatic sequelae, such as cyst hemorrhage, flank pain, recurrent infections, nephrolithiasis, and symptoms of mass effect (i.e., early satiety, nausea and vomiting, and abdominal discomfort), from their enlarged kidneys. ${ }^{89}$ Consequently, patients with ADPKD are often required to undergo native nephrectomy when these symptoms become intractable or when, in the course of preparing for renal transplantation, the native kidneys are found to impinge upon the true pelvis and preclude the placement of a donor allograft. ${ }^{9-11}$ Additionally, native nephrectomy may be undertaken in the presence of suspected malignancy; RCC is 2 to 3 times more likely in the ADPKD-ESRD population than in the ESRD population without ADPKD. ${ }^{12}$ Although the indications for native nephrectomy may be related to kidney size, the decision to proceed with native nephrectomy is often undertaken on an individual basis, without specific reference to kidney size measurements.

The goal of our study was to determine if pre-transplant maximal kidney length (MKL) - an objective size criterion readily available in a clinical setting - can predict eventual nephrectomy status in ADPKD patients undergoing renal transplantation. Furthermore, we evaluate whether augmenting this measure by adjusting for height (HtMKL), weight (WtMKL) or BMI (BMI-MKL), could enhance our ability to predict eventual nephrectomy status. Specifically, we sought to determine if the need for future native nephrectomy could be confidently ruled in or ruled out at the time of transplant assessment. 


\section{Methods}

This is a single-centre, cohort study conducted at the London Health Sciences Centre, London, Canada. Institutional Ethics approval was obtained (REB \#11-117).

\section{Data collection}

We identified all adult ADPKD patients who underwent renal transplantation at our institution between January 1, 2000 and December 31, 2012. Chart review was undertaken to determine patient demographic information, transplantation characteristics, nephrectomy status, timing and indication, pre-transplant MKL, date of first dialysis and total follow-up time. MKL was obtained from pre-transplant ultrasonographic investigations, as well as from computed tomography (CT) scans in rare instances where the former were unavailable $(n=2)$. HtMKL, WtMKL and BMI-MKL were calculated from the data collected.

\section{Statistical analysis}

The variables of interest - demographics, donor type, time on dialysis, MKL and associated measures, timing of MKL measurements and follow-up time - were compared across the Nephrectomy and No-nephrectomy groups using the twotailed Mann-Whitney $U$ test (ordinal data) and Fisher's Exact test (nominal data). Statistical software was used to construct receiver operator characteristic (ROC) curves for the continuous variables of interest (MKL, HtMKL, WtMKL, BMI-MKL) as predictors of the binary outcome of Nephrectomy and the No-nephrectomy groups. The point with the highest Youden index on each ROC curve was used to determine optimal cut-off values. The McNemar Chi-Squared Test was used to compare the sensitivities and specificities of optimal cut-off points on separate ROC curves. The Holm-Bonferonni correction method ${ }^{13}$ was applied to maintain the family-wise error rate at $\alpha=0.05$. All $p$ values retaining their significance post-correction are indicated throughout the paper with an asterisk (*). All statistical calculations were performed with the IBM SPSS 20 and MedCalc 12 statistical packages.

\section{Results}

A total of 102 patients with ADPKD underwent renal transplantation at our institution between January 1, 2000 and December 31, 2012. In all, 84 patients $(82.4 \%)$ met all of our eligibility criteria. The remaining 18 patients $(17.6 \%)$ were excluded from further analysis due to missing or incomplete records $(n=13)$, remote native nephrectomy prior to the study period $(n=3)$, or ongoing consideration for post-transplant native nephrectomy at the time of data collection $(\mathrm{n}=2)$.

\begin{tabular}{|c|c|c|c|}
\hline \multirow{2}{*}{ Parameter } & \multicolumn{2}{|c|}{ N (\%)/Median (IQR) } & \multirow{2}{*}{$p$ value } \\
\hline & Nephrectomy & AUS & \\
\hline Age (yr) & $50(41-57)$ & 54 (IOR $47-62$ ) & 0.104 \\
\hline Sex & 6.4 & 6.4 & 0.91 \\
\hline Male & $33(49 \%)$ & $11(65 \%)$ & 0.289 \\
\hline Female & $34(51 \%)$ & $6(35 \%)$ & 1.00 \\
\hline BMI $\left(\mathrm{kg} / \mathrm{m}^{2}\right)$ & $27.0(24.3-29.8)$ & 26.9 (IOR 23.8-29.7) & 0.947 \\
\hline $\begin{array}{l}\text { Time spent on dialysis } \\
\text { (days before transplant) }\end{array}$ & $424(31-745)$ & 507 (IOR 186-1094) & 0.289 \\
\hline Donor type & 11 & 4 & 0.40 \\
\hline LD & $7(18 \%)$ & $12(41 \%)$ & 0.121 \\
\hline NDD & $8(69 \%)$ & $46(47 \%)$ & 0.71 \\
\hline DCD & $2(13 \%)$ & $9(12 \%)$ & 0.48 \\
\hline Kidney size & 5 & 2 & 0.71 \\
\hline MKL (cm) & 27.0 (IOR 24.6-35.1) & 19.7 (IOR 18.0-23.0) & $<0.001^{*}$ \\
\hline $\mathrm{HtMKL}(\mathrm{cm} / \mathrm{m})$ & 16.8 (IOR 14.3-18.4) & 11.6 (IOR 10.3-13.1) & $<0.001^{*}$ \\
\hline WtMKL $(\mathrm{cm} / \mathrm{kg})$ & 0.37 (IQR 0.29-0.43) & 0.25 (IQR 0.22-0.31) & $<0.001^{*}$ \\
\hline BMI-MKL $\left(\mathrm{cm} / \mathrm{kg} / \mathrm{m}^{2}\right)$ & 1.0 (IQR 0.91-1.30) & 0.73 (IOR 0.63-0.86) & $<0.001^{*}$ \\
\hline $\begin{array}{l}\text { Time of kidney size measurements } \\
\text { (days before transplant) }\end{array}$ & 471 (IOR 135-1140) & 446 (IQR 320-633) & 0.819 \\
\hline Follow-up post-transplant (days) & 1627 (IOR 625-2786) & 1828 (IOR 728-2727) & 0.776 \\
\hline
\end{tabular}

BMI: body mass index; LD: living donor; NDD: neurologic determination of death, DCD: donation after cardiac death, IQR: inter-quartile range; MKL: maximal kidney length; HtMKL: height MKL; WtMKL: weight MKL. * $p$ values retaining their significance post-Holm-Bonferonni correction. 
Cristea et al.

\section{Nephrectomy incidence, timing and indication}

A total of 17 patients (20.2\%) underwent native nephrectomy during the study period; 14 of these patients underwent a single operation with either bilateral or unilateral kidney removal, while 3 underwent staged procedures, for a total of 20 separate procedures. The most common indication for native nephrectomy was solely symptomatic in nature $(\mathrm{n}=$ 11 ), followed by allograft space concerns with or without associated symptoms $(n=7)$, and the presence of suspicious complex cysts $(n=2)$. Symptoms in our cohort included flank pain, abdominal discomfort, early satiety, nausea and vomiting, bleeding cysts and recurrent infection.

\section{Comparison between Nephrectomy and No-nephrectomy groups}

No significant differences were found between the 2 groups with respect to demographic characteristics, time spent on dialysis, donor sources, or median follow-up posttransplant (Table 1). The median MKL of the Nephrectomy group $(27.0 \mathrm{~cm}$; interquartile range [IQR] 24.6-35.1) was significantly greater than that of the No-nephrectomy group (19.7 cm; IQR 18.0-23.0), ( $\left.p<0.001^{*}\right)$. HtMKL, WtMKL and $\mathrm{BMI}-\mathrm{MKL}$ revealed a similar relationship between the groups. MKL of the native kidneys was measured a median of 459 days (IQR 318-637) prior to transplantation, with no significant difference noted between the groups. Ultrasonographic investigations were used to determine MKL in $82(97.7 \%)$ patients; CT scans were used in the remaining $2(2.3 \%)$.

\section{Predictive value of MKL and associated measures}

ROC curves constructed for the MKL, HtMKL, WtMKL and $B M I-M K L$ variables revealed no significant differences between the respective areas under the curve (AUC) (Fig. 1). The optimal cut-off point on the MKL ROC curve was $>21.5 \mathrm{~cm}$, which corresponds to a sensitivity of $94.1 \%(95 \%$ $\mathrm{Cl} 71.3-99.9)$ and a specificity of $70.1 \%(95 \% \mathrm{Cl} 57.7-80.7)$ for the eventual nephrectomized state. Figure 2 illustrates

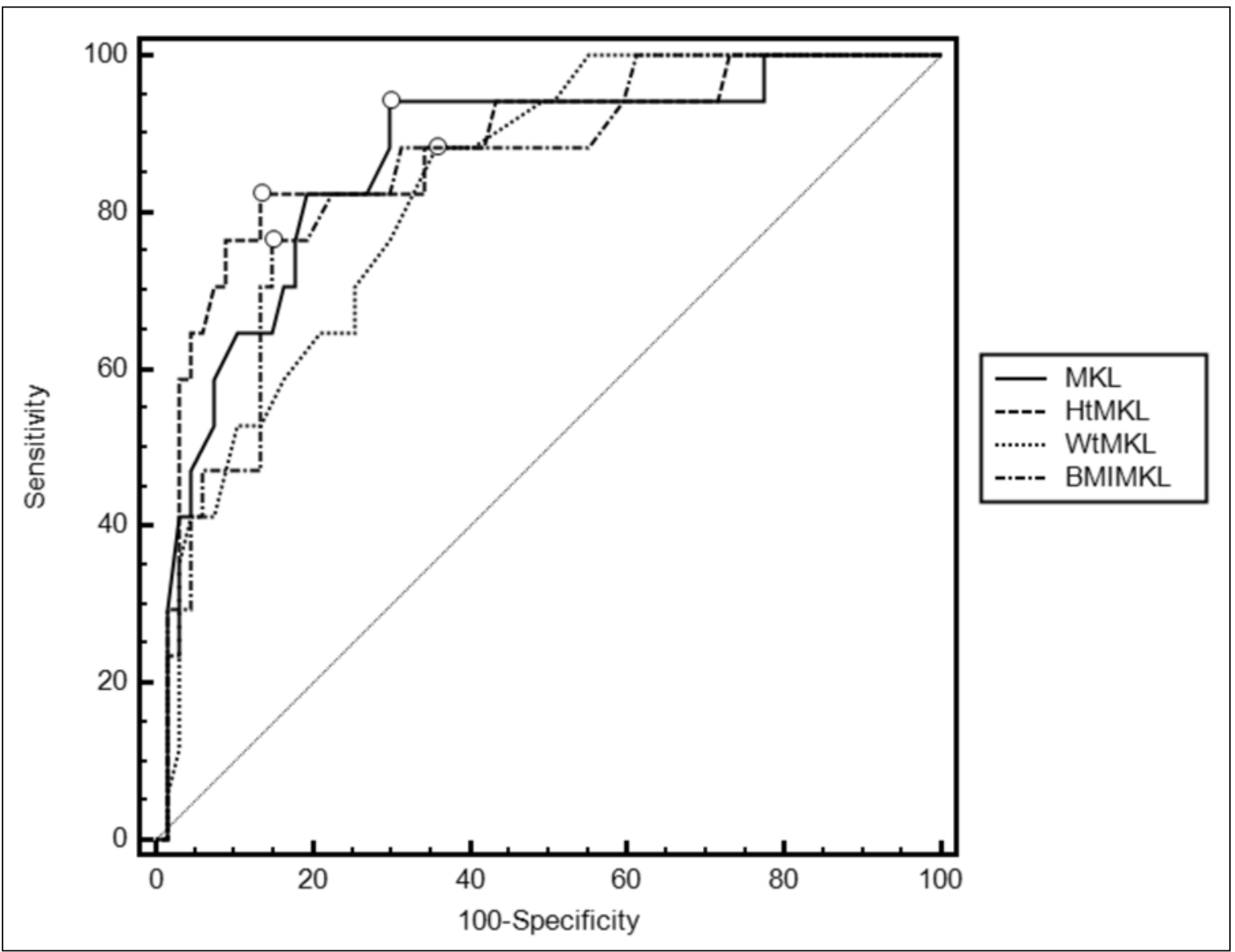

Fig. 1. Receiver operating characteristic (ROC) curves for maximal kidney length (MKL) and associated measures, as predictors of eventual nephrectomized state; optimal cut-off points are indicated with a circle on each curve. Areas under the curve (AUC) as follows: MKL 0.867 (95\% Cl 0.775-0.931; $p<0.001 \%$ ); Height MKL 0.878 (95\% Cl 0.788-0.939; $p<0.001 *$ ); Weight MKL 0.831 (95\% Cl $0.734-$ $\left.0.904 ; p<0.001^{*}\right)$; Body mass index MKL 0.847 (95\% CI 0.752-0. $915 ; p<0.001 *$ ). AUC did not differ significantly in pair-wise comparisons [data not shown]. All $p$ values indicated with a ${ }^{*}$ retained their significance post-Holm-Bonferonni correction. 


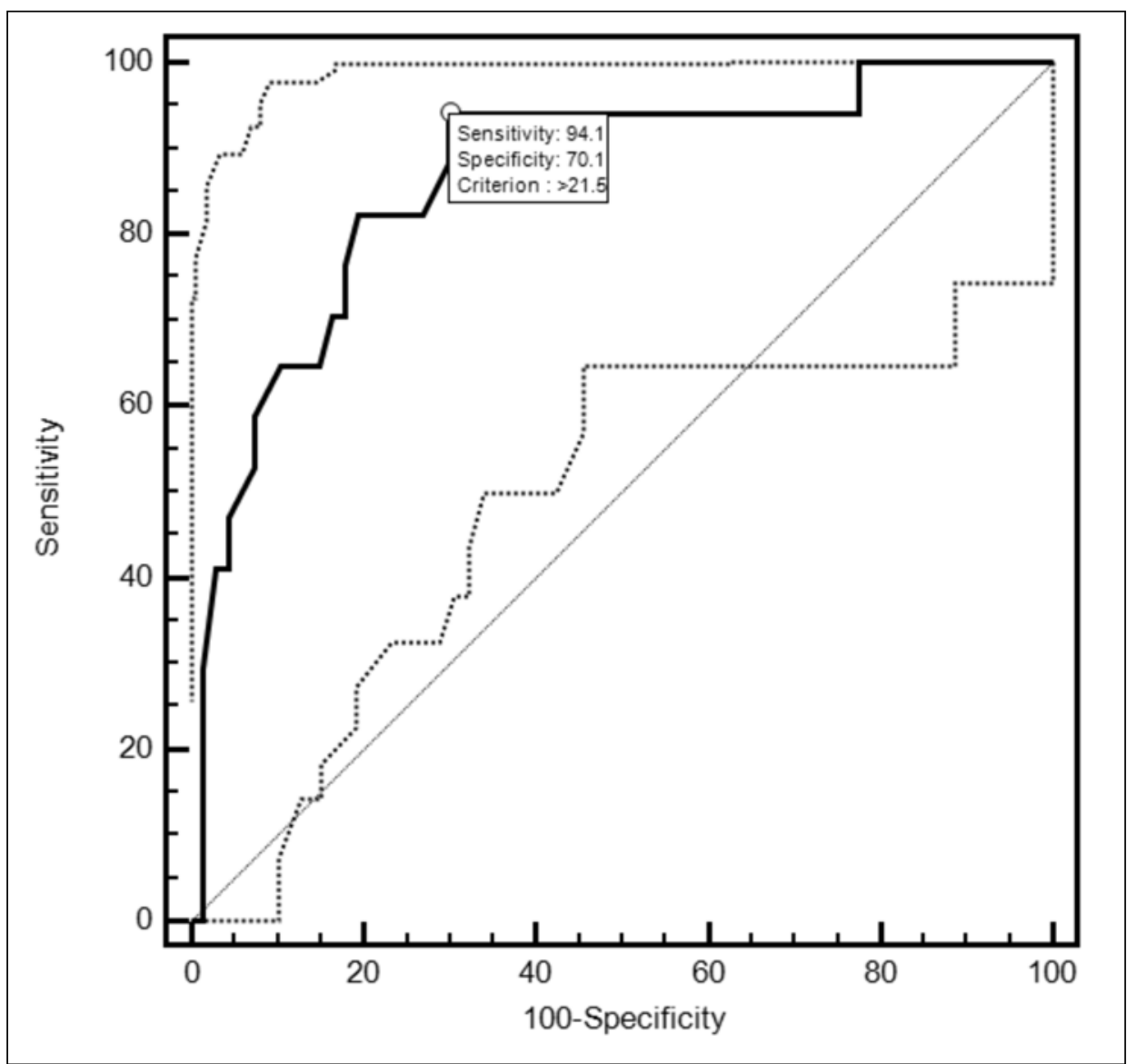

Fig. 2. Receiver operating characteristic (ROC) curve with $95 \% \mathrm{Cl}$ for maximal kidney length (MKL), as a predictor of eventual nephrectomized state; optimal cut-off point with associated values is indicated. Area under the curve (AUC) is $0.867(95 \% \mathrm{Cl} 0.775-0.931 ; p<0.001 *)$. For a cut-off point of $>21.5 \mathrm{~cm}$, the sensitivity is $94.1 \%$ (95\% Cl 71.3-99.9), specificity is 70.1\% (95\% CI 57.7-80.7), positive likelihood ratio 3.15 (95\% Cl 2.14-4.64), negative likelihood ratio $0.08(95 \% \mathrm{Cl} 0.01-0.57)$. All $p$ values indicated with a * retained their significance postHolm-Bonferonni correction.

the MKL ROC curve with its optimal cut-off point and 95\% confidence intervals. The HtMKL ROC curve yielded an optimal cut-off point of $>13.9 \mathrm{~cm} / \mathrm{m}$, which corresponds to a sensitivity of $82.4 \%(95 \% \mathrm{Cl} 56.6-96.2)$ and a specificity of $86.6 \%$ (95\% Cl 76.9-93.7) for the eventual nephrectomized state. Further analysis revealed that the $16.6 \%$ increase in specificity offered by the optimal cut-off point on the HtMKL curve was statistically significant $\left(p<0.001^{*}\right)$. The corresponding $11.7 \%$ decrease in sensitivity on the HtMKL curve did not reach significance $(p=0.5)$.

\section{Discussion}

Native nephrectomies performed on our cohort of patients were undertaken in similar frequencies prior to, concurrent with, or post-transplantation, indicating the lack of any particular timing preference at our institution during the study period. Most nephrectomies were performed for symptomatic reasons alone (55\%); abdominal pain and cyst hem- orrhage were the most frequently cited complaints, in keeping with the literature. ${ }^{2}$

Overall, our results reveal that pre-transplant MKL is a strong predictor of eventual nephrectomy status, with an ROC AUC of 0.867 and an optimal sensitivity of $94.1 \%$ and specificity of $70.1 \%$ for the eventual nephrectomized state, corresponding to a cut-off criterion of $>21.5 \mathrm{~cm}$. This would appear to suggest that, in a clinical setting, native nephrectomy may not be required for a substantial number of patients whose MKL is less than $21.5 \mathrm{~cm}$. In fact, only 1 patient in our cohort with an MKL $<21.5 \mathrm{~cm}$ underwent native nephrectomy, secondary to the presence of a suspected malignancy and in the absence of any other symptoms.

Although the natural history of ADPKD involves a general increase in kidney size over time, recent data suggest that native kidneys may, in fact, be subject to a significant (up to $40 \%$ ) decrease in overall volume post-transplantation, even in the absence of a significant mammalian target of rapamycin (mTOR) inhibitor. ${ }^{14,15}$ Although a concomitant increase in liver volume was noted by the study in question, ${ }^{14} \mathrm{a}$ decrease in the native kidney volume may nonetheless substantially decrease the risk of symptom based renal complications. This decrease may render durable our efforts to rule out the need for native nephrectomy early in the transplant assessment process since, if native kidneys can be expected to shrink post-transplantation, a pre-transplant determination of MKL is likely to represent a lifetime maximum for a patient's overall kidney size.

Of note, the optimal-cut off point on the HtMKL ROC curve of $>13.9 \mathrm{~cm} / \mathrm{m}$, with a sensitivity of $82.4 \%$ and a specificity of $86.6 \%$ for the eventual nephrectomized state, significantly enhanced specificity $(+16.5 \%)$ as compared to $M K L$ alone. The corresponding decrease in sensitivity $(-11.7 \%)$ did not reach statistical significance; however, it is likely that our cohort was simply underpowered to determine even moderate differences in sensitivity, since this measure depends upon the relative performance of the two tests with respect to our [small] Nephrectomy group $(\mathrm{n}=$ 17). While the increase in specificity offered by the HtMKL 
Cristea et al.

measure represents an improvement over MKL alone, it should be noted that the overall performance of the test was not improved, as evidenced by respective ROC AUCs that did not significantly differ (Fig. 1). Furthermore, the high threshold required to proceed with native nephrectomy casts doubt on the utility of this measurement to contribute anything of substance to the decision-making process over MKL alone. While a pre-transplant kidney size measurement may be used to reassure a patient that they are unlikely to ever require a native nephrectomy, it is more difficult to envision such a measurement being used as a rationale to proceed with native nephrectomy in the absence of clinical symptoms, the latter of which are already sufficient to prompt a consideration of native nephrectomy. Thus, an increase in specificity for the eventual nephrectomy state may be of limited clinical utility, especially if it comes at the potential expense of sensitivity. Nevertheless, it is plausible that in the setting of a more accurate measure of kidney size, such as total kidney volume (TKV), a measure adjusting for height (htTVK) may further enhance the specificity for eventual nephrectomy state to the point of yielding some clinical utility. However, at present such measures are not routinely used in clinical settings. ${ }^{3}$

The limitations of our study include its single-centre retrospective design. The former is noteworthy because the threshold to perform a native nephrectomy has been influenced by the patient population and practice patterns at our centre. To validate our findings, a multicentre analysis would be of use to increase the number of events (nephrectomy) and remove surgical bias. A further shortcoming of our study is the use of a limited measurement of kidney size $(\mathrm{MKL})$ in lieu of measurements, such as TKV, that are known to be more robust. While we acknowledge this disadvantage, MKL was chosen because it was the simplest and most consistently recorded measure at our institution. However, it is worth noting that while there is considerable variability between sonographic measurements, kidney length is the most reproducible measurement using this imaging technique, insofar as measurements of the kidney are concerned. ${ }^{16}$ Nevertheless, future studies using an estimate of TKV may well realize an enhanced predictive power given that these are generally estimated on the basis of magnetic resonance imaging or CT scans where measurements are considerably more reliable.

\section{Conclusion}

Our data suggest that pre-transplant measurements of MKL can predict eventual nephrectomy status. To our knowledge, we are the first to quantitatively describe this relationship. In a clinical setting, MKL may be used to confidently rule out the need for future native nephrectomy in a subset of patients, and may thus guide patient conversations to the effect of lending more weight to the decision to leave the native kidneys in situ.

Competing interests: Dr. Cristea, Dr. Yanko, Dr. Felbel, Dr. House, Dr. Sener and Dr. Luke declare no competing financial or personal interests.

This paper has been peer-reviewed.

\section{References}

1. Gabow PA. Autosomal dominant polycystic kidney disease. N Engl J Med 1993;329:332-42. http:// dx.doi.org/10.1056/NEJM199307293290508

2. Torres VE, Harris $\mathrm{PC}$. Autosomal dominant polycystic kidney disease: the last 3 years. Kidney Int 2009;76:149-68. htrp://dx.doi.org/10.1038/ki.2009.128

3. Steinman TI. Polycystic kidney disease: A 2011 update. Curr Opin Nephrol Hypertens 2012;21:189-94. http://dx.doi.org/10.1097/MNH.0b013e3283501 la7

4. Grantham JJ, Torres VE, Chapman AB, et al. Volume progression in polycystic kidney disease. N Engl J Med 2006;354:2122-30. http://dx.doi.org/10.1056/NEJMoa054341

5. Grantham JJ, Cook LI, Torres VE, et al. Determinants of renal volume in autosomal-dominant polycystic kidney disease. Kidney Int 2008;73:108-16. http://dx.doi.org/10.1038/si.ki.5002624

6. Tokiwa $S$, Muto $S$, China $T$, et al. The relationship between renal volume and renal function in autosomal dominant polycystic kidney disease. Clin Exp Nephrol 2011;15:539-45. http://dx.doi.org/10.1007/ s10157-011-0428-y

7. Chapman $A B$, Bost JE, Torres VE, et al. Kidney volume and functional outcomes in autosomal dominant polycystic kidney disease. Clin J Am Soc Nephrol 2012;7:479-86. http://dx.doi.org/10.2215/ CJN.09500911

8. Alam A, Perrone RD. Management of ESRD in patients with autosomal dominant polycystic kidney disease. Adv Chronic Kidney Dis 2010;17:164-72. http://dx.doi.org/10.1053/i.ackd.2009.12.006

9. Wagner MD, Prather JC, Barry JM. Selective, concurrent bilateral nephrectomies at renal transplantation for autosomal dominant polycystic kidney disease. J Urol 2007;177:2250-4; discussion 2254. http:// dx.doi.org/10.1016/i.juro.2007.01.146

10. Fuller TF, Brennan TV, Feng $S$, et al. End stage polycystic kidney disease: Indications and timing of native nephrectomy relative to kidney transplantation. J Urol 2005;174:2284-8. http://dx.doi. org/10.1097/01.ju.0000181208.06507.an

11. Cohen D, Timsit MO, Chretien Y, et al. Place of nephrectomy in patients with autosomal dominant polycystic kidney disease waiting for renal transplantation. Prog Urol 2008; 18:642-9. http://dx.doi. org/10.1016/i.purol.2008.06.004

12. Haij $P$, Ferlicot $S$, Massoud $W$, et al. Prevalence of renal cell carcinoma in patients with autosomal dominant polycystic kidney disease and chronic renal failure. Urology 2009;74:631-4. http://dx.doi. org/10.1016/j.urology.2009.02.078

13. Holm S. A simple sequentially rejective multiple test procedure. Scand I Stat 1979;6:65.

14. Yamamoto $\mathrm{T}$, Watarai $Y$, Kobayashi $\mathrm{T}$, et al. Kidney volume changes in patients with autosomal dominant polycystic kidney disease after renal transplantation. Transplantation 2012;93:794-8. http://dx.doi. org/10.1097/TP.0b013e318246f910

15. Goldfarb DA. Re: Kidney volume changes in patients with autosomal dominant polycystic kidney disease after renal transplantation. J Urol 2012;188:2268. http://dx.doi.org/10.1016/i.juro.2012.07.074

16. O'Neill WC, Robbin ML, Bae KT, et al. Sonographic assessment of the severity and progression of autosomal dominant polycystic kidney disease: the Consortium of Renal Imaging Studies in Polycystic Kidney Disease (CRISP). Am J Kidney Dis 2005;46:1058-64. http://dx.doi.org/10.1053/i.ajkd.2005.08.026

Correspondence: Dr. Patrick Luke, Department of Surgery, Division of Urology, Western University, University Hospital, LHSC, 339 Windermere Rd, London, 0N N6A 5A5; patrick.luke@lhsc.on.ca 